\title{
THE CONJUGACY PROBLEM AND CYCLIC HNN CONSTRUCTIONS
}

\author{
LEIF LARSEN
}

(Received 7 November 1975; revised 16 August 1976)

\begin{abstract}
We consider two related classes of groups. For any group $G$ of the first of these - the smallest class containing all finitely generated free groups and closed under cyclic amalgamations - we show that for any $U, W \in G$ we can "effectively describe" the set of all $x$ and $y$ with $U^{x}$ and $W^{y}$ conjugate in $G$. For the second class, which consists of all groups obtained from the first class through cyclic $H N N$ constructions, we solve the conjugacy and power-conjugacy problems.
\end{abstract}

\section{Introduction}

We use the notation

$$
G^{*}=\left\langle G, t ; t S_{1} t^{-1}=S_{-1}\right\rangle
$$

only for cyclic $H N N$ constructions. Thus, in (1) we assume $G$ to be a given group, $t$ a letter not occurring in $G$, and $S_{1}, S_{-1}$ elements of the same order in $G$. If $G$ has a presentation $G=\langle A ; B\rangle$, then $G^{*}$ can be presented by

$$
G^{*}=\left\langle A, t ; B, t S_{1} t^{-1}=S_{-1}\right\rangle .
$$

In this paper we consider the conjugacy problem for two closely related classes of groups. The first is the smallest class $\mathscr{A}$ containing all finitely generated (henceforth f.g.) free groups and closed under cyclic amalgamations. The second class $\mathscr{A}^{*}$ consists of all cyclic $H N N$ constructions

$$
G^{*}=\left\langle G, t ; t S_{1} t^{-1}=S_{-1}\right\rangle
$$

with $G \in \mathscr{A}$. Since all groups in $\mathscr{A}$ must be torsion-free, it suffices to require $S_{1}$ and $S_{-1}$ to be both non-trivial or both trivial here. Of course, if $S_{1}, S_{-1}=1$, then $G^{*} \in \mathscr{A}$ as well.

This work was supported in part by an AFOSR Grant. 
For each pair of elements $U, W$ of the group $G$, let $C P_{G}(U, W)$ denote the set of conjugate powers of $U$ and $W$ in $G$; that is,

$$
C P_{G}(U, W)=\left\{(x, y) \in \mathbf{Z}^{2} ; U^{x} \underset{G}{ } W^{y}\right\}
$$

where " $G$ " denotes the conjugacy relation in $G$. If $U^{x}$ and $W^{y}$ are non-trivial for some $(x, y) \in C P_{G}(U, W)$, then $U$ and $W$ are said to be power-conjugate in $G$.

To describe the sets $C P_{G}(U, W)$ and some other subsets of $Z^{2}$, we make use of the notation

$$
(r, s)+(a, b) \mathbf{Z}=\left\{(r+a x, s+b x) \in \mathbf{Z}^{2} ; x \in \mathbf{Z}\right\}
$$

for any $r, s, a, b \in \mathbf{Z}$. In particular, we write just $(a, b) \mathbf{Z}$ for this set if $r, s=0$.

We can now state some of our results.

THEOREM 1. All groups in $\mathscr{A}$ and $\mathscr{A}^{*}$ have solvable generalized word problem with respect to cyclic subgroups.

The trivial subgroup is cyclic, hence all groups in $\mathscr{A}$ and $\mathscr{A}^{*}$ have solvable word problem.

Throughout this paper, by "compute" we mean "effectively compute".

THEOREM 2. For any non-trivial $U, W \in G \in \mathscr{A}$ we can compute integers $a, b, c_{1}, \cdots, c_{n}$ such that

$$
C P_{G}(U, W)=(a, b) \mathbf{Z} \cup\left[\bigcup_{i=1}^{n}\left(a c_{i},-b c_{i}\right) \mathbf{Z}\right]
$$

From these two theorems it follows that all groups in $\mathscr{A}$ have solvable conjugacy and power-conjugacy problems.

Using a certain embedding of free products with amalgamation into related $H N N$ groups, as constructed by Miller \& Schupp (1973), we deduce Theorem 2 from

Theorem 3. If $U, W \in G^{*}=\left\langle G, t ; t S_{1} t^{-1}=S_{-1}\right\rangle \in \mathscr{A}^{*}$ are non-trivial and $S_{1}$ and $S_{-1}$ are not power-conjugate in $G$, then we can compute integers $a, b, c_{1}, \cdots, c_{n}$ such that

$$
C P_{G} \cdot(U, W)=(a, b) Z \cup\left[\bigcup_{i=1}^{n}\left(a c_{i},-b c_{i}\right) \mathbf{Z}\right]
$$

For arbitrary groups in $\mathscr{A}^{*}$ we only establish

Theorem 4. All groups in $\mathscr{A}^{*}$ have solvable conjugacy and powerconjugacy problems.

Note that free products of f.g. groups with f.g. amalgamations, even with the factors free, can have unsolvable conjugacy problem, see Miller (1971). 
Thus, to solve the conjugacy problem for such groups we need restrictions such as: the factors free with cyclic subgroups amalgamated, Lipschutz (1966); the factors free with the amalgamating map extending to an isomorphism of the factors, Larsen (1976); or as in the present work. Negative results like the above also exist for $H N N$ groups: for example by the Miller-Schupp embedding.

Our results generalize those of Anshel \& Stebe (1974) who proved that the groups studied by Lipschutz (1966) have solvable power-conjugacy problem, and also, if $G$ is such a group which is residually free and 2 -free (every 2-generator subgroup is free), then $\left\langle G, t ; t S_{1} t^{-1}=S_{-1}\right\rangle$ has solvable conjugacy problem.

Let us also relate our results to those of Pietrowski (1974) about non-cyclic one-relator groups with non-trivial center. Pietrowski proved that such groups can be presented by

$$
G=\left\langle a_{1}, \cdots, a_{m} ; a_{1}^{p_{1}}=a_{2}^{q_{1}}, \cdots, a_{m-1}^{p_{m-1}}=a_{m}^{q_{m-1}}\right\rangle
$$

if $G / G^{\prime}$ is not free abelian of rank two, and by

$$
G=\left\langle a, a_{1}, \cdots, a_{m} ; a a_{1} a^{-1}=a_{m}, a_{1}^{p_{1}}=a_{2}^{q_{1}}, \cdots, a_{m-1}^{p_{m-1}}=a_{m-1}^{q_{m-1}}\right\rangle
$$

otherwise. In both these we have $p_{i}, q_{i} \geqq 2$ for each $i$, hence, the first defines a group in $\mathscr{A}$ and the latter a group in $\mathscr{A}^{*}$.

The author has benefited from the referee's suggestion of using the Miller-Schupp embedding to obtain these results more directly than in the original.

\section{Some definitions and notation}

We only consider groups defined by specific presentations. This allows us to represent elements of any group $G$ by freely reduced words on the generators of $G$ and their inverses. As notation for such words we use upper case Roman letters in the range $R, \cdots, W$, together with the special symbol $\Lambda$ for the empty word. We also use 1 to denote the identity in arbitrary groups.

If $U, W \in G$, then $U=W$ means $U$ and $W$ define the same element of (the abstract group) $G$, while $U \equiv W$ means $U$ and $W$ are identical as words. The cyclic subgroup of $G$ generated by $U$ is denoted by $\langle U\rangle$.

We use

$$
\left(G_{1} * G_{2} ; R_{1}=R_{2}\right)
$$

to denote the free product of $G_{1}$ and $G_{2}$ with the cyclic subgroups $\left\langle R_{1}\right\rangle$ and $\left\langle R_{2}\right\rangle$ amalgamated via $R_{1} \mapsto R_{2}$. The elements $R_{1} \in G_{1}$ and $R_{2} \in G_{2}$ are of course assumed to be of the same order. 
Let us now characterize $\mathscr{A}$ as the union

$$
\mathscr{A}=\bigcup_{k \geq 0} \mathscr{A}_{k}
$$

where $\mathscr{A}_{0}$ is the class of f.g. free groups, and for every $k \geqq 1, G \in \mathscr{A}_{k}$ if and only if

$$
G=\left(G_{1} * G_{2} ; R_{1}=R_{2}\right)
$$

for some $R_{i} \in G_{i} \in \mathscr{A}_{k_{i}}$ with $k_{i}<k$ for $i=1,2$. This gives us a means of proving results about $\mathscr{A}$ by induction on $k$.

Before proving results about cyclic $H N N$ constructions

$$
G^{*}=\left\langle G, t ; t S_{1} t^{-1}=S_{-1}\right\rangle,
$$

let us establish some terminology about elements of such groups. Now, let $W \in G^{*}$ be a given word. If neither $t$ nor $t^{-1}$ occurs in $W$, then $W$ is called $t$-free. If $W$ is not $t$-free, then

$$
W \equiv W_{0} t^{\varepsilon_{1}} W_{1} \cdots t^{\varepsilon_{n}} W_{n}
$$

where each $W_{i}$ is $t$-free. Here, and throughout this paper, we use $\varepsilon, \varepsilon^{\prime}, \varepsilon_{1}$, etc., to denote \pm 1 . The number of $t$-symbols in $W \in G^{*}$ is called the $t$-length of $W$ and denoted by $l_{i}(W)$. Thus, if $W$ is given by (2), then $l_{t}(W)=n$. If $W \in G^{*}$ contains no subword $t^{\varepsilon} W_{i} t^{-\varepsilon}$ with $W_{i} t$-free and belonging to $\left\langle S_{\varepsilon}\right\rangle$, then $W$ is called $t$-reduced. If all cyclic permutations of $W$ are $t$-reduced, and $W$ is either $t$-free or begins with a $t$-symbol, then $W$ is called cyclically $t$-reduced. Thus, if the $W$ in (2) is cyclically $t$-reduced, then $W_{0} \equiv \Lambda$. We also say that $U, W \in G^{*}$ are $t$-parallel if they contain identical sequences of $t$-exponents.

\section{Conjugate powers and cyclic $H N N$ constructions}

The following result is valid for any group $G$.

Lemma 5. Let $U, V, W \in G$ with $\langle V\rangle$ infinite, and suppose that $U^{i} W^{-i} \in\langle V\rangle$ for all $i \geqq 0$. Then $U^{2} \neq W^{2}$ implies $U^{x} \neq W^{x}$ for all $x \neq 0$.

Proof. If $U V^{a} U^{-1}=V^{b}$ implies $a, b=0$, then $U=W$; otherwise $U^{2} W^{-2}=U\left(U W^{-1}\right) U^{-1}\left(U W^{-1}\right)$ and $U W^{-1}$ cannot both belong to $\langle V\rangle$. Hence, from $U^{2} \neq W^{2}$ it follows that $U V^{a} U^{-1}=V^{b}$ for some minimal $a>0$.

In part for later use, let us prove

Claim. Let $z$ be given and set $q=b / a$. Then, if $U^{i} V^{z} W^{-i}=V^{z_{i}}$ for every $i \geqq 1$, it follows that

$$
z_{i}-z=\left(1+q+\cdots+q^{i-1}\right)\left(z_{1}-z\right) .
$$


We establish this claim by induction on $i$. For $i=1$ it is obvious, so suppose the result for $i$ and consider

$$
V^{z_{i+1}}=U^{i+1} V^{z} W^{-i-1}=U V^{z_{i}} W^{-1}=U V^{z_{i}-z} U^{-1} U V^{z} W^{-1} .
$$

This in turn implies that

$$
V^{z_{i+1}-z_{1}}=U V^{z_{i}-z} U^{-1},
$$

hence, $z_{i+1}-z_{1}=k b=q\left(z_{i}-z\right)$ where $z_{i}-z=k a$. Thus,

$$
z_{i+1}-z=z_{1}-z+q\left(z_{i}-z\right)=\left(1+q+\cdots+q^{i}\right)\left(z_{1}-z\right) \text {. }
$$

In the proof of the present lemma we have $z=0$ and $z_{i}=$ $\left(1+q+\cdots+q^{i-1}\right) z_{1}$ with $z_{1} \neq 0$. From $U^{x}=W^{x}$ with $x>0$ it now follows that $z_{x}=0$. But then

$$
1-q^{x}=(1-q)\left(1+q+\cdots+q^{x-1}\right)=0
$$

as well, yielding $q=-1$ since $q=1$ is impossible. Since $q=-1$ yields $z_{2}=0$, we must have $U^{x} \neq W^{x}$ for all $x \neq 0$ if $U^{2} \neq W^{2}$.

Using Lemma 5 we easily prove

Proposition 6. If $U, W \in G^{*}=\left\langle G, t ; t S_{1} t^{-1}=S_{-1}\right\rangle$ are not conjugate to elements of $G$, and $\left\langle S_{1}\right\rangle,\left\langle S_{-1}\right\rangle$ are infinite, then there exist integers $a, b$, and $c$ with

$$
C P_{G} \cdot(U, W)=(a, b) \mathbf{Z} \cup(a c,-b c) \mathbf{Z} .
$$

Proof. We may assume $U$ and $W$ to be cyclically $t$-reduced, and let $a^{\prime}, b^{\prime}>0$ be minimal with $U^{a^{\prime}}$ and $W^{b^{\prime}}$ of the same $t$-length. By Collins (1969, Lemma 3) it follows that if $(x, y) \in C P_{G} \cdot(U, W)$, then $|x| l_{l}(U)=|y| l_{t}(W)$. Thus, if $(x, y) \in C P_{G} \cdot(U, W)$, then $(|x|,|y|) \in\left(a^{\prime}, b^{\prime}\right) \mathbf{Z}$. It now suffices to prove: If $\left(a^{\prime}, \varepsilon b^{\prime}\right) \notin C P_{G} \cdot(U, W)$, yet $(x, y) \in C P_{G} \cdot(U, W)$ with $x y=$ $\varepsilon|x y| \neq 0$, then

$$
(x, y) \in\left(2 a^{\prime}, 2 \varepsilon b^{\prime}\right) \mathbf{Z} \subseteq C P_{G} \cdot(U, W) .
$$

To this end, suppose that $x>0$ and $y=\varepsilon|y| \neq 0$. Hence, $x=a^{\prime} z$ and $y=\varepsilon b^{\prime} z$ for some $z>0$. Now, if $U^{x}=V W^{y} V^{-1}$, then $U_{0}^{z}=W_{0}^{z}$ where $U_{0} \equiv U^{a^{\prime}}$ and $W_{0}=V W^{e b^{\prime}} V^{-1}$ is cyclically $t$-reduced. Since this implies that $U_{0}^{i} W_{0}^{-i} \in\left\langle S_{-\varepsilon}\right\rangle$ for every $i \geqq 0$ where $U \equiv t^{e} U^{\prime}$, it follows that $U_{0}^{2}=W_{0}^{2}$. Moreover, from the assumption that $\left(a^{\prime}, \varepsilon b^{\prime}\right) \notin C P_{G^{*}}(U, W)$ it follows that $U_{0} \neq W_{0}$, hence $z$ must be even.

For $U, W \in G^{*}=\left\langle G, t ; t S_{1} t^{-1}=S_{-1}\right\rangle$ and $U^{\prime}, W^{\prime} \in G$ we now simply write $C P_{*}(U, W)$ for $C P_{G} \cdot(U, W)$ and $C P\left(U^{\prime}, W^{\prime}\right)$ for $C P_{G}\left(U^{\prime}, W^{\prime}\right)$.

Since $C P_{*}\left(S_{1}, S_{1}\right)=C P_{*}\left(S_{i}, S_{j}\right)$ for every $i, j= \pm 1$, the following result is easily deduced from Britton's Lemma (1963). 
LemMa 7. If $G^{*}=\left\langle G, t ; t S_{1} t^{-1}=S_{-1}\right\rangle$, with $(x, y) \in C P\left(S_{i}, S_{j}\right)$ implying $|x|=|y|$ for every $i, j= \pm 1$, then

$$
C P_{*}\left(S_{1}, S_{1}\right)=\bigcup_{i, j= \pm 1} C P\left(S_{i}, S_{j}\right)
$$

As a technical aid we need

LEMMA 8. Let $G^{*}=\left\langle G, t ; t S_{1} t^{-1}=S_{-1}\right\rangle$ and suppose that $(x, y) \in C P\left(S_{i}, S_{i}\right)$ implies $|x|=|y|$ for $i= \pm 1$. Now, if $(x, y) \in C P *\left(S_{1}, S_{1}\right)$ and $V \in G^{*}$ is of minimal $t$-length such that $S_{1}^{x}=V S_{1}^{y} V^{-1}$, then $V$ can contain at most one subword of the form $t^{-*} V_{0} t^{*}$ with $V_{0} t$-free.

Proof. Since $t^{-\varepsilon} V_{o} t^{\varepsilon} S_{\varepsilon}^{z} t^{-\varepsilon} V_{0}^{-1} t^{\varepsilon}=S_{\varepsilon}^{z^{\prime}}$ implies $|z|=\left|z^{\prime}\right|$, such subwords of $V$ are only needed to change the sign of powers $S_{\varepsilon}^{z}$ occurring in the $t$-reductions of $V S_{1}^{y} V^{-1}$. Clearly, at most one such change is necessary.

The following result plays an important role in the proof of Theorem 4 .

LEMmA 9. If $G^{*}=\left\langle G, t ; t S_{1} t^{-1}=S_{-1}\right\rangle, \quad(a, b) \in C P\left(S_{1}, S_{-1}\right) \quad$ with $|a| \neq|b|$, all $(x, y) \in C P\left(S_{1}, S_{-1}\right)$ satisfy $(|x|,|y|) \in(|a|,|b|) Z$, and $d=$ $\operatorname{gcd}(a, b)$ with $a=k d$ and $b=m d$, then

(1) $\left(k^{n} d, m^{n} d\right) \in C P_{*}\left(S_{1}, S_{1}\right)$ for all $n \geqq 1$;

(2) if $(x, y) \in C P_{*}\left(S_{1}, S_{1}\right)$ with $|x| \neq|y|$, then $(|x|,|y|)$ or $(|y|,|x|)$ belongs to $\left(|k|^{n} d,|m|^{n} d\right) \mathbf{Z}$ for some $n \geqq 1$;

(3) if $(x,-x) \in C P_{*}\left(S_{1}, S_{1}\right)$, then $(z,-z) \in C P\left(S_{1}, S_{1}\right) \cup C P\left(S_{-1}, S_{-1}\right)$ for some $z$ with $z=x$ or one of $(x, z)$ and $(z, x)$ in $\left(k^{n} d, m^{n} d\right) \mathbf{Z}$ for some $n \geqq 1$.

Proof. Note that the condition on $C P\left(S_{1}, S_{-1}\right)$ implies that all $(x, y) \in$ $C P\left(S_{1}, S_{1}\right) \cup C P\left(S_{-1}, S_{-1}\right)$ satisfy $|x|=|y|$.

(1) This follows by induction on $n$ since for every $n \geqq 0$ we have $\left(k^{n+1} d, m^{n+1} d\right)=\left(k^{n} a, m^{n} b\right)$.

(2) We prove by induction on $l_{t}(V)$ that for every $i, j= \pm 1$ : if $S_{i}^{x}=$ $V S_{j}^{y} V^{-1}$ with $|x| \neq|y|$, then the conclusion of (2) holds. The result is obvious for $V t$-free, so suppose that $V \equiv V^{\prime} t^{\varepsilon} V_{0}$ with $V_{0} t$-free and the result established for $V^{\prime}$. From

$$
S_{i}^{x}=V^{\prime} t^{\varepsilon} V_{0} S_{j}^{y} V_{0}^{-1} t^{-\varepsilon}\left(V^{\prime}\right)^{-1}=V^{\prime} t^{\varepsilon} S_{\varepsilon}^{z} t^{-\varepsilon}\left(V^{\prime}\right)^{-1}=V^{\prime} S_{-\varepsilon}^{z}\left(V^{\prime}\right)^{-1}
$$

we then get either $|x|=|z|$ with $|z| \neq|y|,|x| \neq|z|$ with $|z|=|y|$, or $|x| \neq|z|$ and $|z| \neq|y|$. Only the last of these need further investigation, and by symmetry, the following two cases suffice:

CASE 1. $(|x|,|z|)=\left(|k|^{n} d f,|m|^{n} d f\right)$ and $(|z|,|y|)=(|k| d g,|m| d g)$ for some $f, g \in \mathbf{Z}$. Since $|k| d g=|m|^{n} d f$ and $\operatorname{gcd}(k, m)=1$, it follows that 
$f=|k| f^{\prime} \quad$ and $\quad g=|m|^{n} f^{\prime} . \quad$ Thus, $\quad(|x|,|y|)=\left(|k|^{n} d f,|m| d g\right)=$ $\left(|k|^{n+1} d f^{\prime},|m|^{n+1} d f^{\prime}\right)$.

CASE 2. $(|z|,|x|)=\left(|k|^{n} d f,|m|^{n} d f\right) \quad$ and $\quad(|z|,|y|)=(|k| d g,|m| d g)$. From $|k|^{n} d f=|k| d g$ we now get $g=|k|^{n-1} f$. Thus, $(|y|,|x|)=$ $\left(|m| d g,|m|^{n} d f\right)=\left(|k|^{n-1} d|m| f,|m|^{n-1} d|m| f\right)$. Since $|x| \neq|y|$, we must have $n-1 \geqq 1$.

Before continuing, let us establish

Claim. If $t$ only occurs with exponent $\varepsilon$ in $W$ and $l_{f}(W)=n \geqq 1$, then $S_{j}^{x}=W S_{j}^{y} W^{-1}$ implies that $(|x|,|y|) \in\left(|k|^{n} d,|m|^{n} d\right) \mathbf{Z}$ if $\varepsilon=1$, and $(|y|,|x|) \in\left(|k|^{n} d,|m|^{n} d\right) \mathbf{Z}$ if $\varepsilon=-1$.

We use induction on $n$. Now, if $W \equiv W^{\prime} t^{\varepsilon} W_{0}$ with $W_{0} t$-free, then

$$
S_{j}^{x}=W^{\prime} t^{\epsilon} W_{0} S_{j}^{y} W_{0}^{-1} t^{\varepsilon}\left(W^{\prime}\right)^{-1}=W^{\prime} t^{\varepsilon} S_{\varepsilon}^{z} t^{-\varepsilon}\left(W^{\prime}\right)^{-1}=W^{\prime} S_{-\varepsilon}^{z}\left(W^{\prime}\right)^{-1}
$$

for some $z$. If $j=\epsilon$, then $|z|=|y|$ and

$$
S_{-j}^{x}=t^{\varepsilon} S_{j}^{x} t^{-\varepsilon}=t^{\epsilon} W^{\prime} S_{-j}^{z}\left(t^{\varepsilon} W^{\prime}\right)^{-1}
$$

with $l_{t}\left(t^{\varepsilon} W^{\prime}\right)=l_{t}(W)$. Thus, we need only consider $j=-\varepsilon$, and hence $(z, y) \in C P\left(S_{-,}, S_{j}\right)$. If $n=1$ we are done, and otherwise $(|x|,|z|) \in$ $\left(|k|^{n-1} d,|m|^{n-1} d\right) \mathbf{Z}$ if $\varepsilon=1$, and $(|z|,|x|) \in\left(|k|^{n-1} d,|m|^{n-1} d\right) \mathbf{Z}$ if $\varepsilon=-1$. Since $j=-\varepsilon$, the claim now follows from case 1 of (2).

Instead of (3), let us prove: If $S_{i}^{x}=V S_{i}^{-x} V^{-1}$ for $i= \pm 1$, then there exists a $z$ as asserted in (3). We use induction on $l_{t}(V)$ where $V$ is assumed to be of minimal such length; this makes Lemma 8 applicable. If $V$ is $t$-free, then we can take $z=x$, so suppose that $V$ is not $t$-free, and that the result holds for all $V^{\prime}$ with $l_{t}\left(V^{\prime}\right)<l_{i}(V)$. By Lemma 8 we can write $V \equiv U^{-1} W$ where $t$ does not occur with exponent $-\varepsilon$ in $U$ or $W$. Thus, we now get $U S_{i}^{\times} U^{-1}=$ $W S_{i}^{-x} W^{-1}$ where all $t$ 's can be removed by $t$-reductions on both sides. It easily follows from the above claim that $U$ or $W$ cannot be $t$-free. Suppose now that $(x,-x)$ does not belong to $C P\left(S_{1}, S_{1}\right) \cup C P\left(S_{-1}, S_{-1}\right)$. Then write $U \equiv U^{\prime} t^{\varepsilon} U_{0}$ and $W \equiv W^{\prime} t^{\epsilon} W_{0}$ with $U_{0}$ and $W_{0} t$-free. From $U S_{i}^{x} U^{-1}=W S_{i}^{-x} W^{-1}$ it now follows that $U^{\prime} S_{-\varepsilon}^{y}\left(U^{\prime}\right)^{-1}=W^{\prime} S_{-\varepsilon}^{-y}\left(W^{\prime}\right)^{-1}$ for some $y$. If $\varepsilon=i$, then $x=y$ and the inductive hypothesis applies. Suppose therefore that $\varepsilon \neq i$. By symmetry, it suffices to consider $\varepsilon=-1$. Thus, $(x, y) \in C P\left(S_{1}, S_{-1}\right)$ with $(x,-y) \notin C P\left(S_{1}, S_{-1}\right)$ implies that $(x, y) \in(k d, m d) Z$. If now $(y,-y) \in$ $C P\left(S_{i}, S_{i}\right)$ for $i= \pm 1$, then we are done. But otherwise the inductive hypothesis implies that $(y, z)$ or $(z, y)$ belongs to $\left(k^{n} d, m^{n} d\right) \mathbf{Z}$ with $n \geqq 1$ for some $(z,-z) \in C P\left(S_{1}, S_{1}\right) \cup C P\left(S_{-1}, S_{-1}\right)$. By Case 2 of (2) and the minimality of $l_{t}(V)$, it follows that $(y, z) \in\left(k^{n} d, m^{n} d\right) Z$. But then, by Case 1 of (2) we get $(x, z) \in\left(k^{n+1} d, m^{n+1} d\right) \mathbf{Z}$. 


\section{Collins' Lemma and cyclic $H N N$ constructions}

Let $U$ and $W$ be cyclically $t$-reduced elements of the HNN group

$$
G^{*}=\left\langle G, t ; t S_{1} t^{-1}=S_{-1}\right\rangle
$$

with $U \equiv t^{\epsilon} U^{\prime}$. By Collins' Lemma we then know that $U$ and $W$ are conjugate in $\mathrm{G}^{*}$ if and only if

$$
U=S_{-\varepsilon}^{x} W_{\pi} S_{-\varepsilon}^{-x}
$$

for some $x$ and cyclic permutation $W_{\pi}$ of $W$. Thus, if we can decide for any $T, U, W \in G^{*}$ whether or not any $x$ satisfies

$$
U T^{\mathrm{x}} W=T^{\mathrm{x}}
$$

then we can solve the conjugacy problem for all cyclically $t$-reduced elements of $G^{*}$ that are not $t$-free.

To pursue the above reasoning in a more general setting, let $H$ and $K$ be subgroups of some group $G, U$ and $W$ elements of $G$, and consider

$$
\{(S, T) \in H \times K ; U S W=T \text { (in } G)\}
$$

as a subset of the group $H \times K$ (the direct product). Since this is the graph of the function $G \rightarrow G$, given by $V \leftrightarrow U V W$, restricted to the (possibly empty) subset of $H$ mapped into $K$, we use the notation

$$
\operatorname{gph}(U, W ; H, K)=\{(S, T) \in H \times K ; U S W=T\} .
$$

For any subgroup $N$ of $H \times K$ and element $\left(S_{0}, T_{0}\right) \in H \times K$, let $N\left(S_{0}, T_{0}\right)$ denote the right translate of $N$ by $\left(S_{0}, T_{0}\right)$. Noting that $\operatorname{gph}(U, W ; H, K)$ is a subgroup of $H \times K$ if and only if $W=U^{-1}$, we get

LEMMA 10. If $\left(S_{0}, T_{0}\right) \in g p h(U, W ; H, K)$, then

$$
\operatorname{gph}(U, W ; H, K)=\left[\operatorname{gph}\left(U, U^{-1} ; H, K\right)\right]\left(S_{0}, T_{0}\right) .
$$

Proof. Since $U S_{0} W=T_{0}$, it follows that $U S W=T$ if and only if $U S W\left(U S_{0} W\right)^{-1}=U S S_{0}^{-1} U^{-1}=T T_{0}^{-1}$. Hence, $(S, T) \in \operatorname{gph}(U, W ; H, K)$ if and only if $\left(S S_{0}^{-1}, T T_{0}^{-1}\right) \in \operatorname{gph}\left(U, U^{-1} ; H, K\right)$; that is, if and only if $(S, T) \in$ $\left[\operatorname{gph}\left(U, U^{-1} ; H, K\right)\right]\left(S_{0}, T_{0}\right)$.

In this paper we only consider cyclic graphs $\operatorname{gph}\left(U, W ; C_{1}, C_{2}\right)$; that is, graphs where the subgroups $C_{1}$ and $C_{2}$ are cyclic. Moreover, if $C_{1}=\left\langle T_{1}\right\rangle$ and $C_{2}=\left\langle T_{2}\right\rangle$ are infinite, then we identify these with $\mathbf{Z}$ through $T_{i}^{x} \mapsto x$, and simplify our notation by setting

$$
\operatorname{gph}\left(U, W ; T_{1}, T_{2}\right)=\left\{(x, y) \in \mathbf{Z}^{2} ; U T_{1}^{x} W=T_{2}^{y}\right\} .
$$


The following result is now immediate.

Corollary 11. If $T_{1}, T_{2} \in G$ are of infinite order and $(r, s) \in$ $\operatorname{gph}\left(U, W ; T_{1}, T_{2}\right)$, then

$$
\operatorname{gph}\left(U, W ; T_{1}, T_{2}\right)=(r, s)+(a, b) \mathbf{Z}
$$

where $(a, b) \mathbf{Z}=\operatorname{gph}\left(U, U^{-1} ; T_{1}, T_{2}\right)$.

In view of this corollary we say that cyclic graphs can be (effectively) computed in $G$ if for any $U, W, T_{1}, T_{2} \in G$ we can:

(1) compute the orders of $T_{1}$ and $T_{2}$;

(2) list all pairs of $\operatorname{gph}\left(U, W ;\left\langle T_{1}\right\rangle,\left\langle T_{2}\right\rangle\right)$ if $\left\langle T_{1}\right\rangle$ or $\left\langle T_{2}\right\rangle$ is finite;

(3) compute $r, s, a, b \in \mathbf{Z}$ with $(a, b) \mathbf{Z}=\operatorname{gph}\left(U, U^{-1} ; T_{1}, T_{2}\right)$ and $U T_{1}^{r} W=T_{2}^{s}$ if and only if $g p h\left(U, W ; T_{1}, T_{2}\right) \neq \varnothing$ whenever $\left\langle T_{1}\right\rangle$ and $\left\langle T_{2}\right\rangle$ are infinite.

The following lemma is our major technical result concerning computability of cyclic graphs.

Lemma 12. If we can compute cyclic graphs in $G$, then we can also compute such graphs in the $H N N$ group $G^{*}=\left\langle G, t ; t S_{1} t^{-1}=S_{-1}\right\rangle$.

Proof. From the hypothesis it follows that $G$ has solvable generalized word problem with respect to cyclic subgroups. Thus, we can effectively $t$-reduce and cyclically $t$-reduce elements of $G^{*}$; hence, by changing $U$ and $W$ if necessary we may assume that $T_{1}$ and $T_{2}$ are cyclically $t$-reduced, $U$ and $W t$-reduced.

Now, if $T_{1}$ is $t$-free while $T_{2}$ is not, then $U T_{1}^{x} W=T_{2}^{y}$ implies that

$$
|y| l_{t}\left(T_{2}\right) \leqq l_{t}(U)+l_{t}(W) .
$$

For each such $y$ we can $t$-reduce $U^{-1} T_{2}^{y} W^{-1}$ to obtain a $t$-reduced word $V_{y}$, and then check if $V_{y} \in\left\langle T_{1}\right\rangle$ in $G$. Such a $y$ is necessarily unique, and by hypothesis we can determine the corresponding $T_{1}^{x}=V_{y}$. The case with the roles of $T_{1}$ and $T_{2}$ interchanged is similar.

Two cases remain.

CASE 1. $T_{1}, T_{2} \in G$. Unless $U$ and $W^{-1}$ are $t$-parallel we get $\operatorname{gph}\left(U, W ;\left\langle T_{1}\right\rangle,\left\langle T_{2}\right\rangle\right)=\varnothing$. Suppose that $U$ and $W^{-1}$ are $t$-parallel and proceed by induction on $l_{t}(U)=l_{t}(W)$. If $U$ and $W$ are $t$-free the hypothesis of the lemma applies, so suppose that $U \equiv U^{\prime} t^{\varepsilon} U_{0}$ and $W \equiv W_{0} t^{-\varepsilon} W^{\prime}$ with $U_{0}$ and $W_{0} t$-free. Moreover, assume that $g p h\left(U^{\prime}, W^{\prime} ;\left\langle T_{1}^{\prime}\right\rangle,\left\langle T_{2}^{\prime}\right\rangle\right)$ can be computed for any $T_{1}^{\prime}, T_{2}^{\prime} \in G$. Assume also that $T_{1}, T_{2}, S_{1}$, and $S_{-1}$ are of infinite order; otherwise the result is easily obtained.

If now 


$$
U^{\prime} t^{\varepsilon} U_{0} T_{1}^{x} W_{0} t^{-\varepsilon} W^{\prime}=T_{2}^{y}
$$

for some $x$ and $y$, then we must have

$$
\operatorname{gph}\left(U_{0}, W_{0} ; T_{1}, S_{\varepsilon}\right)=\left(r_{0}, s_{0}\right)+\left(a_{0}, b_{0}\right) \mathbf{Z}
$$

and

$$
\operatorname{gph}\left(U^{\prime}, W^{\prime} ; S_{-\varepsilon}, T_{2}\right)=\left(r^{\prime}, s^{\prime}\right)+\left(a^{\prime}, b^{\prime}\right) \mathbf{Z}
$$

where all constants can be computed. Moreover, unless $a_{0}, a^{\prime} \neq 0$, it is easy to compute $g p h\left(U, W ; T_{1}, T_{2}\right)$. But clearly, if

$$
\operatorname{lcm}\left(\left|b_{0}\right|,\left|a^{\prime}\right|\right)=m_{0} b_{0}=m^{\prime} a^{\prime}>0,
$$

then we must have

$$
\operatorname{gph}\left(U, U^{-1} ; T_{1}, T_{2}\right)=\left(m_{0} a_{0}, m^{\prime} b^{\prime}\right) \mathbf{Z} .
$$

It now remains to check if $U T_{1}^{r} W=T_{2}^{s}$ for any $r, s$ with $0 \leqq r<\left|m_{0} a_{0}\right|$.

CASE 2. $\quad l\left(T_{i}\right)=k_{\mathrm{i}}>0$ with $\operatorname{lcm}\left(k_{1}, k_{2}\right)=m_{i} k_{i}$ for $i=1,2$. Then, for any non-trivial $(x, y) \in \operatorname{gph}\left(U, U^{-1} ; T_{1}, T_{2}\right)$ we must have

$$
|x| k_{1}=|y| k_{2}=z m_{1} k_{1}=z m_{2} k_{2}
$$

for some $z>0$. From this we get $x=\varepsilon_{1} z m_{1}$ and $y=\varepsilon_{2} z m_{2}$ for some $\varepsilon_{1}$ and $\varepsilon_{2}$. Writing $T_{0}=U T_{1}^{\varepsilon_{1} m_{1}} U^{-1}$ and $T_{3}=T_{2}^{\varepsilon_{2} m_{2}}$ with $T_{0}$ and $T_{3}$ cyclically $t$-reduced and $T_{0} \equiv t^{\varepsilon} T_{0}^{\prime}$, it follows from $U T_{1}^{x} U^{-1}=T_{2}^{y}$ that $T_{0}^{z}=T_{3}^{z}$. Hence, for all $i \geqq 0$ we must have $T_{0}^{i} T_{3}^{-i} \in\left\langle S_{-\varepsilon}\right\rangle$. If now $\left\langle S_{-\varepsilon}\right\rangle$ is infinite, then Lemma 5 shows that we can take $z=2$. If instead the order of $S_{-\varepsilon}$ equals $c<\infty$, then we can bound $z$ by $c$. To see this, note that $T_{0}^{i} T_{3}^{-i}=T_{0}^{j} T_{3}^{-j}$ implies $T_{0}^{i-j}=T_{3}^{i-j}$.

We have thus reduced this case to the subcase where at most one pair $x_{l}$, $x_{2}$ can satisfy $U T_{1}^{x_{1}} W=T_{2}^{x_{2}}$. But such a pair $x_{1}, x_{2}$ exists if and only if a pair $x_{1}^{\prime}, x_{2}^{\prime} \geqq 0$ exists such that

$$
\left(U T_{1}^{r_{1}}\right)\left(T_{1}^{\varepsilon_{1} m_{1}}\right)^{x_{1}}\left(W T_{2}^{-r_{2}}\right)=\left(T_{2}^{\varepsilon_{2} m_{2}}\right)^{x_{2}^{\prime}}
$$

where $x_{i}=\varepsilon_{i} m_{i} x_{i}^{\prime}+r_{i}$ with $o \leqq r_{i}<m_{i}$ for $i=1$,2. Since only finitely many quadruples $\left(\varepsilon_{1}, \varepsilon_{2}, r_{1}, r_{2}\right)$ need be considered, it suffices to show how we can bound $x_{1}^{\prime}, x_{2}^{\prime} \geqq 0$ given that $\varepsilon_{1}, \varepsilon_{2}, m_{1}, m_{2}=1$, and hence $r_{1}, r_{2}=0$.

We now assume that $k_{1}=k_{2}$ with $g p h\left(U, W ; T_{1}, T_{2}\right) \neq \varnothing$ if and only if $U T_{1}^{x} W=T_{2}^{y}$ for some $x, y \geqq 0$. It suffices to bound $x$, so suppose $x$ is large enough so that $U T_{1}^{x} W$ can be $t$-reduced to yield $U^{\prime} T_{1}^{x^{\prime}} W^{\prime}$ as a $t$-reduced word with $x^{\prime}>1$. If $T_{1} \equiv t^{\varepsilon} T_{1}^{\prime}$, then $T_{2}^{-y} U^{\prime} T_{1}^{x^{\prime}} W^{\prime}=1$ implies that

$$
T_{3}^{-1} T_{2}^{-y_{1}} U^{\prime}=S_{-z_{*}}^{z_{1}}
$$


for some $T_{3}$ with $T_{2} \equiv T_{3} t^{\varepsilon} T_{3}^{\prime}, y_{1} \geqq 0$, and $z_{1} \in Z$. Also, $U^{\prime} T_{1}^{x^{\prime}} W^{\prime} T_{2}^{-y}=1$ implies that

$$
T_{1} W^{\prime} T_{2}^{-y_{2}} T_{4}^{-1}=S_{-\varepsilon}^{z_{2}}
$$

for some $T_{4}$ with $T_{2} \equiv T_{4}^{\prime} T_{4} \equiv T_{4}^{\prime} t^{\varepsilon} T_{4}^{\prime \prime}, y_{2} \geqq 0$, and $z_{2} \in \mathbf{Z}$. Considering $t$ lengths, it follows that

$$
t^{*} T_{3}^{\prime} T_{2}^{y-\left(y_{1}+y_{2}+2\right)} T_{4}^{\prime} \equiv \bar{T}_{2}^{x^{\prime}-1}
$$

for some cyclically $t$-reduced cyclic permutation $\bar{T}_{2}$ of $T_{2}$. Also, since

$$
S_{-\varepsilon}^{z_{1}} T_{1}^{x^{\prime}-1}=\bar{T}_{1}^{x^{\prime}-1} S_{-\varepsilon}^{z_{1}}
$$

where $\bar{T}_{1}=S_{-\varepsilon}^{z_{1}} T_{1} S_{-\varepsilon}^{-z_{1}}=t^{\varepsilon} S_{\varepsilon}^{z_{1}} T_{1}^{\prime} S_{-\varepsilon}^{-z_{1}}$, it follows that $U^{\prime} T^{x^{\prime}} W^{\prime}=T_{2}^{y}$ if and only if

$$
\bar{T}_{1}^{x^{\prime}-1} S_{-\varepsilon}^{z_{1}+z_{2}}=\bar{T}_{2}^{x^{\prime}-1} .
$$

Writing $z=z_{1}+z_{2}$, it now follows that

$$
\bar{T}_{1}^{i} S_{-\varepsilon}^{z} \bar{T}_{2}^{-i} \in\left\langle S_{-\varepsilon}\right\rangle
$$

for every $i \geqq 0$. If the order of $S_{- \text {e }}$ equals $c<\infty$, then we can bound $x^{\prime}-1$ by c. If $S_{-\varepsilon}$ is of infinite order, then consider

$$
\bar{T}_{1}^{i} S_{-\varepsilon}^{z} \bar{T}_{2}^{-i}=S_{{ }_{-\varepsilon}}^{z_{i}}
$$

as in the claim used in the proof of Lemma 5. If $\bar{T}_{1} S_{-\varepsilon}^{a} \bar{T}_{1}^{-1}=S_{-\varepsilon}^{b}$ implies $a, b=0$, then $x^{\prime}-1 \leqq 1$. Assume therefore that $q=b / a \neq 0$ and

$$
z_{i}-z=\left(1+q+\cdots+q^{i-1}\right)\left(z_{1}-z\right) \text {. }
$$

We seek a minimal $x>0$ with $z_{x}=0$, that is, with

$$
-z=\left(1+q+\cdots+q^{x-1}\right)\left(z_{1}-z\right) \text {. }
$$

If $z=0$, then $x=1$ or 2 works. If $z_{1}-z=0$, then $z=0$, so suppose that $z, z_{1}-z \neq 0$. But then, at most one $x$ can satisfy

$$
1+q+\cdots q^{x-1}=z /\left(z_{1}-z\right) .
$$

To determine this $x$, note that $q=1$ implies $x=z /\left(z_{1}-z\right)$, while $q \neq 1$ implies

$$
q^{x}-1=(q-1) z /\left(z_{1}-z\right) .
$$

Since all the above reductions are clearly effective, and all constants, $x^{\prime}$, $z_{1}, z_{2}, a$, and $b$ can be computed, the result follows.

For the proof of the next proposition we need

LEMMA 13. If we can compute cyclic graphs in $G_{1}$ and $G_{2}$, then we can also compute such graphs in $G_{1} * G_{2}$ (the free product). 
Proof. Any word $U \in G_{1} * G_{2}$ can be written in reduced form

$$
U \equiv U_{1} \cdots U_{n}
$$

that is, with each $U_{i}$ a word in $G_{1}$ or $G_{2}$, and if $n \geqq 2$, then each $U_{i} \neq 1$ and $U_{i}, U_{i+1}$ belong to distinct factors for each $1 \leqq i<n$. Each $U_{i}$ in (3) is called a syllable of $U$, and the number $n$ of these is called the syllable length of $U$. If $U$ is either empty, belong to a factor, or is of even syllable length (as a reduced word), then $U$ is called cyclically reduced.

Suppose now that $U, W, T_{1}, T_{2} \in G_{1} * G_{2}$ and consider the graph $\operatorname{gph}\left(U, W ;\left\langle T_{1}\right\rangle,\left\langle T_{2}\right\rangle\right)$. The case with $\left\langle T_{\mathrm{i}}\right\rangle$ finite for $i=1$ or 2 is trivial, so suppose $T_{1}$ and $T_{2}$ to be of infinite order. Moreover, by our hypothesis we can reduce and cyclically reduce elements of $G_{1} * G_{2}$; hence, by changing $U$ and $W$ if necessary, let us asssume $T_{1}$ and $T_{2}$ to be cyclically reduced with $U$ and $W$ reduced. Now, if exactly one of $T_{1}$ and $T_{2}$ belongs to a factor, then $U T_{1}^{x} W=T_{2}^{y}$ immediately gives a bound on $|x|$ or $|y|$. This is now just like the beginning of the last proof.

Two cases remain.

CASE 1. $T_{1}$ and $T_{2}$ have syllable length one. Write $U \equiv U_{1} \cdots U_{n}$ and $W \equiv W_{1} \cdots W_{m}$ as reduced words. From $U T_{1}^{x} W=T_{2}^{y}$ it now follows that either all of $U, W, T_{1}$, and $T_{2}$ belong to the same factor, thus making our hypothesis applicable, or one of $U_{n} T_{1}^{x}, T_{1}^{x} W_{1}$, and $U_{n} T_{1}^{x} W_{1}$ must be trivial for some $x$. Whether or not such an $x$, which must be unique, exists can be decided; and if it exists, then we can also compute the pair $(x, y)$.

CASE 2. $\quad T_{i}$ has syllable length $k_{i}>1$ for $i=1,2$. It is elementary to determine $a, b \neq 0$ such that $g p h\left(U, U^{-1} ; T_{1}, T_{2}\right) \neq(0,0) Z$ if and only if $\operatorname{gph}\left(U, U^{-1} ; T_{1}, T_{2}\right)=(a, b) Z$. Thus, as in the last proof, we need only consider the case where at most one pair $x, y$ can satisfy $U T_{1}^{x} W=T_{2}^{y}$. Also, we may also here assume that $k_{1}=k_{2}$, and show how we can bound the search for such $x, y \geqq 0$. If $x$ is large enough, then we can reduce $U T_{1}^{x} W$ to obtain a reduced word $U^{\prime} T_{1}^{x^{\prime}} W^{\prime}$ with $x^{\prime}>0$. But then, from $U^{\prime} T_{1}^{x^{\prime}} W^{\prime}=T_{2}^{y}$ it follows that

$$
T_{3}^{-1} T_{2}^{-y_{1}} U^{\prime}=1=W^{\prime} T_{2}^{-y_{2}} T_{4}^{-1}
$$

for some $T_{3}$ and $T_{4}$ with $T_{3} T_{3}^{\prime} \equiv T_{2} \equiv T_{4}^{\prime} T_{4}$. From this we get $T_{1}^{x^{\prime}}=\bar{T}_{2}^{x^{\prime}}$ for some cyclic permutation $\bar{T}_{2}$ of $T_{2}$, which is possible only if $T_{1}=\bar{T}_{2}$. Since these reductions are effective, the result follows.

To show that cyclic graphs can be computed in all $G^{*} \in \mathscr{A}^{*}$, we make use of the Miller-Schupp embedding 


$$
\varphi: G=\left(G_{1} * G_{2} ; R_{1}=R_{2}\right) \rightarrow G_{0}^{*}=\left\langle G_{0}, t ; t R_{1} t^{-1}=R_{2}\right\rangle
$$

where $G_{0}=G_{1} * G_{2}$. This $\varphi$, which is clearly effective, is induced by:

$$
\varphi(U)=t U t^{-1} \text { for all } U \in G_{1}
$$

and

$$
\varphi(W)=W \text { for all } W \in G_{2} .
$$

Proposition 14. We can compute cyclic graphs in all groups of $\mathscr{A}^{*}$.

Proof. By Lemma 12 it suffices to prove that we can compute cyclic graphs in all $G \in \mathscr{A}$. For this we use induction on $k$ where $G \in \mathscr{A}_{k}$. Since the result is trivial for free groups of rank one, we easily obtain the result for $\mathscr{A}_{0}$ by induction on the rank of f.g. free groups using Lemma 13. For our main inductive step, if the result holds for all $G^{\prime} \in \mathscr{A}_{k^{\prime}}$ with $k^{\prime}<k$, and $G=$ $\left(G_{1} * G_{2} ; R_{1}=R_{2}\right) \in \mathscr{A}_{k}$, then Lemma 13 establishes the result for $G_{0}=$ $G_{1} * G_{2}$, Lemma 12 establishes it for $G_{0}^{*}=\left\langle G_{0}, t ; t R_{1} t^{-1}=R_{2}\right\rangle$, while the Miller-Schupp embedding establishes it for $G$.

Note that this proposition also yields a proof of Theorem 1 . This because $U \in\langle V\rangle$ if and only if $\operatorname{gph}(U, \Lambda ;\langle\Lambda\rangle,\langle V\rangle) \neq \varnothing$.

\section{Conjugate powers and the class $\mathscr{A}^{*}$}

Before turning to the proofs of Theorems 2 and 3, let us establish

Lemma 15. Let $U, W \in G^{*}=\left\langle G, t ; t S_{1} t^{-1}=S_{-1}\right\rangle \in \mathscr{A}^{*}$ be non-trivial and $t$-free where $C P\left(S_{1}, S_{-1}\right)=(0,0) Z$, and assume that Theorem 2 holds for $G$. Then we can compute $a, b, c_{1}, \cdots, c_{n} \in \mathbf{Z}$ such that

$$
C P_{*}(U, W)=(a, b) \mathbf{Z} \cup\left[\bigcup_{i=1}^{n}\left(a c_{i},-b c_{i}\right) \mathbf{Z}\right] .
$$

Proof. If $C P_{*}(U, W) \neq C P(U, W)$, then

$$
U^{x} \widetilde{G}_{i}^{z} S_{i}^{z} S_{j}^{z^{\prime}} \widetilde{G} W^{y}
$$

for some $(x, y) \notin C P(U, W)$, where necessarily $|z|=\left|z^{\prime}\right| \neq 0$ and $i$ and $j$ are uniquely determined by the assumption that $C P\left(U, S_{\mathrm{i}}\right)$ and $C P\left(S_{j}, W\right)$ are $\neq(0,0) \mathbf{Z}$. Since we can compute $C P(U, W)$, we may assume that we have computed

$$
C P\left(U, S_{i}\right)=\left(a_{1}, b_{i}\right) \mathbf{Z} \cup\left[\bigcup_{i=1}^{n_{1}}\left(a_{1} d_{i},-b_{1} d_{i}\right) \mathbf{Z}\right]
$$

and 


$$
C P\left(S_{j}, W\right)=\left(a_{2}, b_{2}\right) Z \cup\left[\bigcup_{i=1}^{n_{2}}\left(a_{2} e_{i},-b_{2} e_{i}\right) \mathbf{Z}\right]
$$

with $a_{1}, a_{2} \neq 0$.

CAse 1. $i \neq j$. Then $C P(U, W)=(0,0) \mathrm{Z}$, and $C P_{*}(U, W)$ can be con structed from $C P\left(U, S_{i}\right)$ and $C P\left(S_{i}, W\right)$ as follows: First, let

$$
\mu=\operatorname{lcm}\left(\left|b_{1}\right|,\left|a_{2}\right|\right)=m_{1} b_{1}=m_{2} a_{2}
$$

and set $(a, b)=\left(m_{1} a_{1}, m_{2} b_{2}\right)$. Next, define $c_{i}$ by

$$
c_{i} \mu=\operatorname{lcm}\left(\left|b_{1} d_{i}\right|,\left|a_{2}\right|\right)
$$

for each $1 \leqq i \leqq n_{1}$, and by

$$
c_{i} \mu=\operatorname{lcm}\left(\left|b_{1}\right|,\left|a_{2} e_{i-n_{1}}\right|\right)
$$

for each $n_{1}+1 \leqq i \leqq n_{1}+n_{2}$. Setting $n=n_{1}+n_{2}$, it easily follows that

$$
C P_{*}(U, W)=(a, b) \mathbf{Z} \cup\left[\bigcup_{i=1}^{n}\left(a c_{i},-b c_{i}\right) \mathbf{Z}\right] .
$$

CASE 2. $i=j$. Suppose that we have computed

and

$$
C P(U, W)=(a, b) \cup\left[\bigcup_{i=1}^{n^{\prime}}\left(a c_{i}^{\prime},-b c_{i}^{\prime}\right) \mathbf{Z}\right]
$$

$$
C P\left(S_{-i}, S_{-i}\right)=(1,1) \cup\left[\bigcup_{i=1}^{n_{0}}\left(f_{i},-f_{i}\right) \mathbf{Z}\right] .
$$

Then, unless $a \neq 0$ and $C P\left(S_{-i}, S_{-i}\right) \neq(1,1) Z$, we must have $C P_{*}(U, W)=$ $C P(U, W)$. To see this, note that if $(x, y) \in C P_{*}(U, W)$ with $(x, y) \notin C P(U, W)$, then

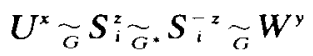

for some $z \neq 0$ with $(z,-z) \in C P\left(S_{-i}, S_{-i}\right)$. Moreover, we must have $(x, z) \in$ $\left(a_{1}, b_{1}\right) \mathbf{Z}, \quad(-z, y) \in\left(a_{2}, b_{2}\right) \mathbf{Z}, \quad$ and $\quad(x,-y) \in(a, b) \mathbf{Z} ;$ otherwise $(x, y) \in$ $C P(U, W)$. Since $\mu=m_{1} b_{1}=m_{2} a_{2}$ as in Case 1 , we must have $\left(m_{1} a_{1}, m_{2} b_{2}\right) \in$ $C P(U, W)$. Moreover, defining $g_{i}$ by

$$
g_{i} \mu=\operatorname{lcm}\left(\left|b_{1}\right|,\left|f_{i}\right|,\left|a_{2}\right|\right)
$$

for each $1 \leqq i \leqq n_{0}$, it follows that

$$
\left(g_{i} m_{1} a_{1},-g_{i} m_{2} b_{2}\right) \in C P_{*}(U, W)
$$

for each such $i$. Now, if the products $a b$ and $m_{1} a_{1} m_{2} b_{2}$ have the same sign, then define $c_{i}^{\prime \prime}$ by 


$$
g_{i} m_{1} a_{1}=c_{i}^{\prime \prime} a
$$

for each $1 \leqq i \leqq n_{0}$. We now get

$$
C P_{*}(U, W)=(a, b) \mathbf{Z} \cup\left[\bigcup_{i=1}^{n}\left(a c_{i},-b c_{i}\right) \mathbf{Z}\right]
$$

where $n=n^{\prime}+n_{0}, c_{i}=c_{i}^{\prime}$ for each $1 \leqq i \leqq n^{\prime}$, and $c_{i}=c_{i-n^{\prime}}^{\prime \prime}$ for each $n^{\prime}+1 \leqq$ $i \leqq n^{\prime}+n_{0}$. If the products $a b$ and $m_{1} a_{1} m_{2} b_{2}$ have opposite signs, then $C P_{*}(U, W)=C P(U, W)$.

As a partial proof of Theorem 3 and 4 we have

Proposition 16. Let $U, W \in G^{*}=\left\langle G, t ; t S_{1} t^{-1}=S_{-1}\right\rangle \in \mathscr{A}^{*}$ be cyclically $t$-reduced and not $t$-free. Then we can compute $a, b, c \in \mathbf{Z}$ such that

$$
C P_{*}(U, W)=(a, b) \mathbf{Z} \cup(a c,-b c) \mathbf{Z} .
$$

Proof. From the proof of Proposition 6, it suffices to prove that we can decide for any cyclically $t$-reduced $U^{\prime}, W^{\prime} \in G^{*}$ with $l_{t}\left(U^{\prime}\right)=l_{t}\left(W^{\prime}\right)>0$ whether or not $U^{\prime} \widetilde{G}^{*} W^{\prime}$. By Collins' Lemma we know that $U^{\prime}$ and $W^{\prime}$ are conjugate in $G^{*}$ if and only if

$$
U^{\prime}=S_{-\varepsilon}^{x} W_{\pi}^{\prime} S_{-\varepsilon}^{-x}
$$

for some $x$ and cyclically $t$-reduced cyclic permutation $W_{\pi}^{\prime}$ of $W^{\prime}$ where $U^{\prime} \equiv t^{\varepsilon} U^{\prime \prime}$. But this is equivalent to having

$$
(x, x) \in \operatorname{gph}\left(U^{\prime},\left(W_{n}^{\prime}\right)^{-1} ; S_{-\varepsilon}, S_{-\varepsilon}\right)
$$

for some $W_{\pi}^{\prime}$. Since only finitely many $W_{\pi}^{\prime}$ need be considered, and

$$
(x, x) \in(r, s)+(a, b) Z
$$

if and only if $(a-b) z=s-r$ for some $z \in \mathbf{Z}$, the result follows from Proposition 14.

We can now complete the

Proof of Theorems 2 and 3. Because of Lemma 15 and Proposition 16 , it suffices to prove Theorem 2 . To prove this theorem for all $G \in \mathscr{A}$ we proceed by induction on $k$ where $G \in \mathscr{A}_{k}$. The result clearly holds for f.g. free groups, so consider $G=\left(G_{1} * G_{2} ; R_{1}=R_{2}\right) \in \mathscr{A}_{k}$ with the result established for $G_{1}$ and $G_{2}$. But then it must obviously hold for $G_{0}=G_{1} * G_{2}$ too. Since the Miller-Schupp embedding

$$
\varphi: G \rightarrow G_{0}^{*}=\left\langle G_{0}, t ; t R_{1} t^{-1}=R_{2}\right\rangle
$$

is easily seen to satisfy

$$
C P_{G}(U, W)=C P_{G_{0}^{*}}(\varphi(U), \varphi(W))
$$


for all $U, W \in G$, the result now follows from Lemma 15 and Proposition 16 . Note that $C P_{G_{0}}\left(R_{1}, R_{2}\right)=(0,0) Z$.

For the proof of Theorem 4 we need

Lemma 17. If $G^{*}=\left\langle G, t ; t S_{1} t^{-1}=S_{-1}\right\rangle \in \mathscr{A}^{*}$, we can decide for any $x$ and $y$ whether or not $(x, y) \in C P_{*}\left(S_{1}, S_{1}\right)$.

Proof. By Theorem 2 we can compute $C P\left(S_{i}, S_{j}\right)$ for each $i, j= \pm 1$. Now, if all $\left(x^{\prime}, y^{\prime}\right) \in C P\left(S_{1}, S_{-1}\right)$ satisfy $\left|x^{\prime}\right|=\left|y^{\prime}\right|$, then Lemma 7 applies. On the other hand, if $C P\left(S_{1}, S_{-1}\right)$ contains some pair $\left(x^{\prime}, y^{\prime}\right)$ with $\left|x^{\prime}\right| \neq\left|y^{\prime}\right|$, then we use Lemma 9. Suppose therefore that $(a, b) \in C P\left(S_{1}, S_{-1}\right)$ satisfies the hypothesis of Lemma 9. Hence, $a=k d$ and $b=m d$ where $d=\operatorname{gcd}(a, b)$.

CASE 1. $|x|=|y|$. Only the subcase with $y=-x$ and $(x,-x) \notin C P\left(S_{i}, S_{i}\right)$ for $i= \pm 1$ need be considered. By Lemma 9, we must determine if $(x, z)$ or $(z, x)$ belongs to $\left(k^{n} d, m^{n} d\right) Z$ for some $n \geqq 1$ with $(z,-z) \in C P\left(S_{1}, S_{1}\right) \cup C P\left(S_{-1}, S_{-1}\right)$. If $(x, z)=\left(k^{n} d f, m^{n} d f\right)$ for some $f \in Z$, then $|f|$ is bounded by $|x|$. Moreover, if $|k| \neq 1$, then $n$ is also bounded. If instead $|k|=1$, then $|m| \neq 1$, and we can easily decide if $\left(m^{n} d f,-m^{n} d f\right) \in$ $C P\left(S_{i}, S_{i}\right)$ for some $n \geqq 1$ and $i= \pm 1$. The result follows by symmetry.

CASE 2. $|x| \neq|y|$. Since $\quad(|x|,|y|) \in\left(|k|^{n} d,|m|^{n} d\right) \mathbf{Z} \quad$ with $(x, y) \notin\left(k^{n} d, m^{n} d\right) \mathbf{Z}$ implies that $(x,-y) \in\left(k^{n} d, m^{n} d\right) \mathbf{Z}$, and $\left(k^{n} d, m^{n} d\right) \mathbf{Z} \subseteq$ $C P_{*}\left(S_{1}, S_{1}\right)$, this case is thus reduced to Case 1 .

We can now prove Theorem 4 .

Proof of Theorem 4. Let $G^{*}=\left\langle G, t ; t S_{1} t^{-1}=S_{-1}\right\rangle \in \mathscr{A}^{*}$, and suppose that $U, W \in G^{*}$ are cyclically $t$-reduced. Now, if exactly one of $U$ and $W$ is $t$-free, then $U$ and $W$ cannot be power-conjugate. If neither of $U$ and $W$ is $t$-free, then Proposition 16 applies.

It remains to consider $U, W \in G$. If $U_{\widetilde{G}} W$, then $U_{\widetilde{G}} \cdot W$. Suppose therefore that $U \underset{G}{+} W$. If still $U \widetilde{G}^{*} W$, then

$$
U \widetilde{G} S_{i \widetilde{G}}^{x} \cdot S_{j \widetilde{G}}^{y} W
$$

for some $x, y$ and $i, j$. By Theorem 2 we can determine all such pairs $(x, y)$, and by Lemma 17 we can decide if $(x, y) \in C P_{*}\left(S_{1}, S_{1}\right)$. This completes the solution of the conjugacy problem for $G^{*}$. Finally, if $U$ and $W$ are power-conjugate in $G$, then they are also power-conjugate in $G^{*}$. Furthermore, if $C P(U, W)=(0,0) \mathrm{Z}$, then $C P_{*}(U, W) \neq(0,0) \mathrm{Z}$ if and only if

$$
U^{x} \underset{G}{\widetilde{G}} S_{i \widetilde{G}^{*}}^{z} S_{j}^{z^{\prime} \widetilde{G}^{*}} W^{y}
$$

for some $x, z, z^{\prime}$, and $y$. But this holds if and only if $C P\left(U, S_{i}\right)$ and $C P\left(S_{i}, W\right)$ are $\neq(0,0) \mathbf{Z}$ for some $i, j= \pm 1$. 


\section{References}

Michael Anshel and Peter Stebe (1974), 'The solvability of the conjugacy problem for certain HNN groups', Bull. Amer. Math. Soc. 80, 266-270.

John L. Britton (1963), 'The word problem', Ann. of Math. (2)77, 16-32.

Donald J. Collins (1969), 'Recursively enumerable degrees and the conjugacy problem', Acta Math. 122, 115-160.

Leif Larsen (1976), 'The solvability of the conjugacy problem for certain free products with amalgamation', J. Algebra, 43, 28-41.

Seymour Lipschutz (1966), 'Generalization of Dehn's result on the conjugacy problem', Bull. Amer. Math. Soc. 17, 759-762.

Wilhelm Magnus, Abraham Karrass, Donald Solitar (1966), Combinatorial Group Theory: Presentations of groups in terms of generators and relators (Pure and Appl. Math. 13. Interscience [John Wiley \& Sons], New York, London, Sydney, 1966).

Charles F. Miller, III (1971), On Group-Theoretic Decision Problems and Their Classification (Annals of Mathematics Studies, 68. Princeton University Press, University of Tokyo Press, Princeton, New Jersey, 1971).

Charles F. Miller, III and Paul E. Schupp (1973), 'The geometry of Higman-Neuman-Neuman extensions', Comm. Pure Appl. Math., 26, 787-802.

Alfred Pietrowski (1974), 'The isomorphism problem for one-relator groups with non-trivial centre', Math. Z. 136, 95-106.

Institute of Math.,

University of Oslo,

Blindern,

Oslo 3, Norway. 